\title{
Lessons Learnt from Collaboratively Creating Maps on a Touch Table
}

\author{
Hien Tran, Craig Anslow, Stuart \\ Marshall, Alex Potanin \\ School of Engineering and Computer \\ Science \\ Victoria University of Wellington \\ \{craig,stuart,alex\}@ecs.vuw.ac.nz
}

\author{
Mairéad de Róiste \\ School of Geography, Environment and \\ Earth Sciences \\ Victoria University of Wellington \\ Mairead.deRoiste@vuw.ac.nz
}

\begin{abstract}
While touch tables have improved support for creative, colocated, collaborative tasks, the very act of studying what groups create on such tables (and how) remains non-trivially difficult. We developed an experimental tool to study what map designs would be created by pairs of users collaborating around a touch table, however to paraphrase the German military strategist Helmuth von Moltke: "no experimental tool survives contact with the table". While running our experiments, we made a series of observations around issues with table interaction, and our initial expectations on how the users would be able to interact with the tool. In this paper, we contribute these observations to assist other researchers considering undertaking a similar course of action.
\end{abstract}

\section{Author Keywords \\ CSCW, map design, touch tables, user studies \\ ACM Classification Keywords}

H.5.2 User Interfaces: Evaluation / Methodology; H.5.2 User Interfaces: Input Devices and Strategies

General Terms

Design, Experimentation

\section{INTRODUCTION}

There already exist user studies that focus on the use of geographic visualization tools to understand the usability, interaction, and geospatial elements of online static and interactive cartographic maps. However, none of the studies have focused on how individual users or groups of users would design online maps if given the opportunity.

As part of a wider research project into this issue of user designed online maps, we created a collaborative map creation tool for use on touch tables. We subsequently ran an experiment to see what kinds of maps teams created, and in doing so made a number of observations around problems and issues that arose due to experimenting with collaboratively creative tools on touch tables.

Permission to make digital or hard copies of all or part of this work for personal or classroom use is granted without fee provided that copies are not made or distributed for profit or commercial advantage and that copies bear this notice and the full citation on the first page. To copy otherwise, or republish, to post on servers or to redistribute to lists, requires prior specific permission and/or a fee.

CHINZ 2011, July 4-5, 2011, Hamilton, New Zealand.

Copyright 2011 ACM 978-1-4503-0676-8/11/07...\$10.00.
These observations serve as this paper's contribution, rather than the actual results of how the final map designs compared. The rest of the paper is organised as follows: to begin we briefly introduce some related work in the wider field of online cartographic studies. Then we introduce the tool - Map Elements Designer (MED) - that forms the basis of our user study, before then describing the user study we undertook. We conclude with our observations and recommendations for other researchers considering running similar user studies on collaboratively creative tools on touch tables.

\section{RELATED WORK}

Traditional cartography relies on both art and rules to communicate geographic information to map users. The movement to an online environment means that users also need to navigate other more interactive map elements or controls. They may need to zoom and pan or even interact with the data themselves online. The user experience of online mapping, therefore, has to contend with a larger number of variables and changing map content.

Increasingly, computer users access a wide variety of maps online. Online map applications can be highly interactive, and they provide an advantage over physical maps as they can also be updated in real time.

Very few tools have looked at exploring the behaviour of users with geospatial data. GIViz [1] is a visualization tool for analysing user interaction behaviour with a geospatial data set. The tool captures the user's mouse behaviour and then superimposes the information as visualizations on the spatial data similar to heat maps. Other visualizations display timing data collected by the tool. This tool was not used with collaborative users or touch tables. Another related technique to employ would be eye tracking [4].

Some researchers have explored the design of touch table displays for co-located information visualization [3] and collaborative design [6]. Others have explored manipulating spatial data combining both hand and feet gestures with a multi-touch wall [5] and a virtual 3D geospatial environment [7]. We are not aware of any user studies that have focused on users collaborating with geospatial applications for multi-touch tables. 


\section{MED: MAP ELEMENTS DESIGNER}

We have developed Map Elements Designer (MED), a tool that enables users to control the placement of controls and cartographic elements for online maps. We had a particular emphasis on how groups of users might collaboratively reach a decision on how a map should look, so we ended up targeting a touch table for displaying MED, as tables are big enough to actively support multiple people working around on the same task. MED consists of two separate components: the map creator and the map design visualizer. In this paper we only present the map creator component since it is the one that fully utilises the touch table technology.

The map creator was developed in ActionScript and runs within the Adobe Flash Player inside a web browser. The component consists of four maps, each with specific map elements for the same geographic location. Instructions are shown at the beginning of each map. When the users proceed, they are presented with an image of the current map, an instructions box on the top right corner, a box (map elements box) containing the different map elements with tooltips providing descriptions for each element (some elements had to be reduced in size to fit inside the box and when dragged outside they enlarge to their original size), and a trash can for discarding unwanted map elements. Figure 1 illustrates some of the maps contained within the map creator.

The users are instructed to drag whichever elements they deem suitable to a desired place on the map, with the option to discard unwanted elements. Once the users are satisfied with their placements and all the elements are either on the map or in the trash can, then they are able to move to the next map. When all four maps are completed, the the data of where each user places the map elements is stored in a database for later analysis.

When displayed on a touch table, only one touch event is processed at a time: the application does not support multiple concurrent actions. This single touch processing ensures that participants work together and negotiate to place a single element, whereas if people can move elements at the same time they may tend to work independently. The buttons and selection points for the map elements were enlarged due to human fingers being the input mechanism [2].

\section{Design Decisions}

Map Creator did not allow users to skip to the next map unless they had placed everything, either on the map or in the trash can. This would ensure that no elements were unintentionally skipped.

The trash can was introduced to force users to think about discarding unwanted elements. A message dialog appears confirming the user's intention of deletion. Trashed elements disappear reducing unwanted clutter. Freeing up space in the elements box would also give the user a sense of progress. The only way to restore the elements from a trash can is to reset all the elements which necessitated that the user has to rethink the placement of all the elements from scratch.

The reset button allowed users to start afresh, when pressed,

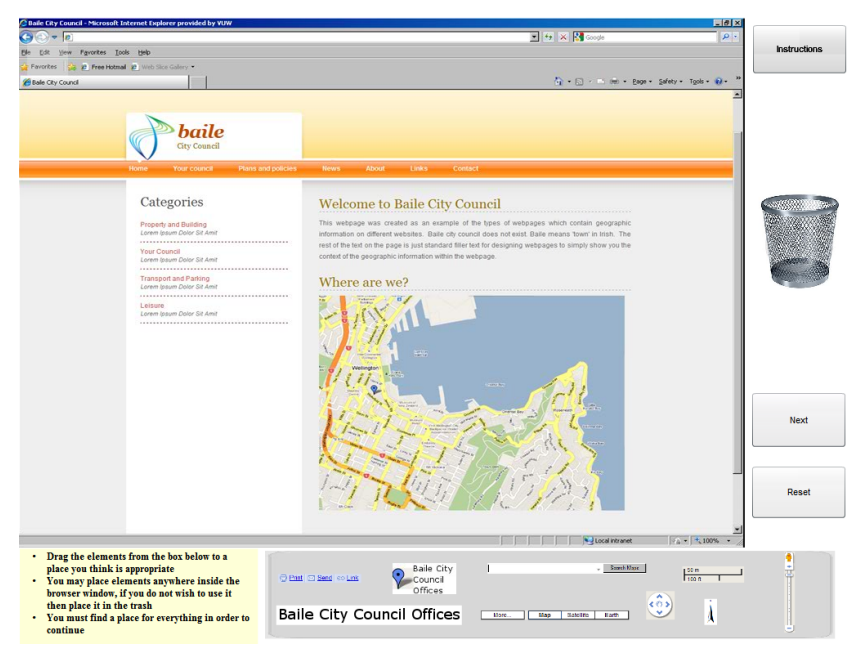

(a) Interactive Map

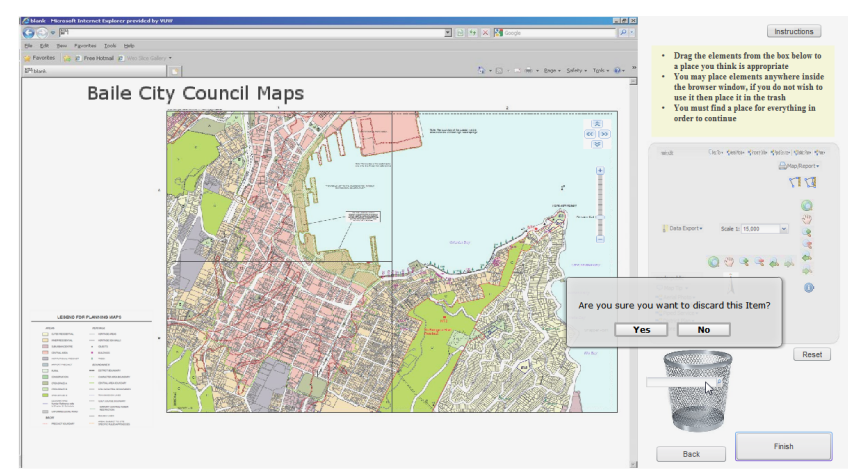

(b) GIS Map (with Discard Confirmation)

Figure 1. Some Map Scenarios

all the elements (including trashed elements) reset back to their original positions in the elements box. We incorporated a back button to allow users to return to previously completed maps and to change or to reflect on their decision.

A brief instructions box was provided to give direction to users that skipped the initial instructions or didn't completely understand it. Furthermore, the descriptive instructions could be reopened with the instructions button.

The elements were positioned randomly on the elements box and aligned neatly so that they would fit inside the box. Any elements that were too large for the box had to be reduced in size, this was especially evident for the last two map scenarios where the map was also a movable element. We did not find any way around this.

The size of the elements were adjusted appropriately to best match the size of the content on the map. Moreover, common sizes used for elements in existing map applications, were best replicated for our elements.

\section{USER STUDY}

In this section, we outline the study we conducted on twelve students (studying either Geographic Information System (GIS) or Computer Science) who used MED in pairs via a touch table. We developed four map scenarios for use in these 
phases, and ran a brief pilot study on MED's user interface to capture and correct any immediate functional problems. Note that the focus of this paper is not on the resulting maps they created, but our observations of any problems they had with our tool or with the touch table.

\section{Map Scenarios}

The four map scenarios used in our experiment ranged in complexity, and in their use of interactive controls. Figure 1 illustrates two of the four different map scenarios.

Interactive Map: The first is a basic interactive map shown in Figure 1(a). The map itself shows a particular geographic location. It also contains a significant portion of low detailed space in the form of a large water body to the top right of the map. The map elements to be placed contain interactive elements from Google Maps, such as a zoom control and a pan control. Users can also place standard map elements such as a north arrow, a scale bar, and map title.

Non-Interactive Map: The second map shows the same map content (with different symbology) but uses a non interactive (standard static image) containing no interactive components. For this map, the user is asked to place the following standard map elements: a legend, map title, north arrow, and scale bar.

PDF Map: The third map is a land use planning map in PDF format which requires more detailed cartographic map elements (for example, a legend may be needed to explain the different symbology) Unlike the first two basic maps where the location on the simulated web page was predetermined, this scenario allows you to move the map around within the web browser, and two layout versions of the same legend map element are provided (the legend may be longer in length or in width).

GIS Map: The final map contains the same content as the previous scenario but is shown as a highly interactive map using online GIS controls displayed inside a blank webpage, see Figure 1(b). Advanced controls such as 'data export' (export spatial data from the map), 'measure' (allows you to measure distances across map) and 'identify' (get information at a particular map location or feature) are included. These controls are similar to the controls provided in a desktop GIS and are available in some online interactive mapping applications. This screenshot also demonstrates the confirmation dialog when a user tries to delete an element.

\section{Procedure}

Our study involved participants working in pairs to complete the map scenarios on a touch table. The participants were observed in our usability lab's controlled environment. The user testing comprised of novice-novice, expert-novice and expert-expert pairings.

The touch table has 1024 by 768 resolution and the screen surface physical size is $570 \mathrm{~mm}$ by $420 \mathrm{~mm}$. The table top stands at $980 \mathrm{~mm}$ height from the floor. The room is dimly lit due to the touch table's sensitivity to light. Two microphones

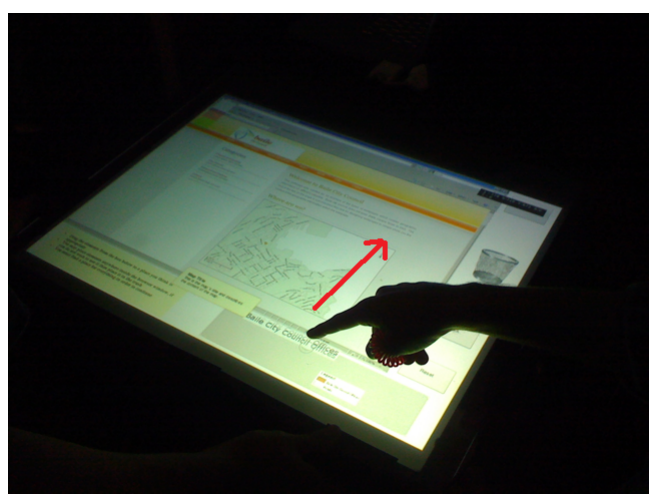

(a) Dragging an element from the elements box.

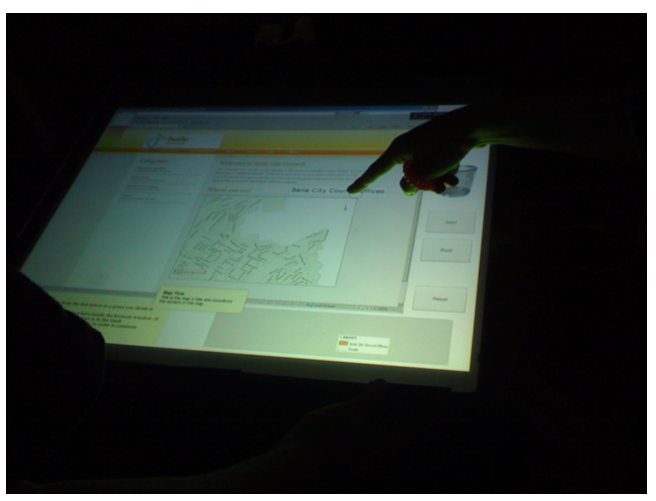

(b) Placing an element on the desired location.

Figure 2. Collaborative Map Elements Design.

are positioned to the side of the table for voice recording and a video camera is elevated to a height pointing fronton towards the table, and rotated to a 45 degrees angle. This records video of the participant's hand movements above the table. Furthermore, a direct screen capture from the touch table is also recorded to see how the application is manipulated by the participants.

At the start of a session, participants are given a simple puzzle program to complete. One person is instructed to wear a red ring signifier on both hands, this is to differentiate between whose hand is which in the video recordings. The puzzle allows the participants to familiarize themselves with the touch table in an informal environment before real testing begins.

\section{RESULTS}

Our observations of the participants using the touch table highlighted a number of issues regarding the usability of the tool, and their behaviour while constructing the final layouts. Figure 2 shows a representative example of dragging objects using our touch table.

Interaction: The vast majority of participants used their index or middle finger to interact with the table. Interestingly, one participant chose to use their thumb, which is not as dexturous or as comfortable as the other fingers. This participant struggled with selecting and moving elements, and their use of the thumb appears to be driven by their selection of which finger to wear the ring on when the experiment began. 
Usability Problems: While the application was single touch, the table itself supported (and could detect) multiple touches. This led to one interesting usability problem, whereby participants might place two fingers on the table, and the application would switch between which finger was controlling the movement of the element. This switch would typically occur if the participant accidentally released pressure on the (currently) controlling finger. In two cases, the second finger (that was not meant to be controlling movement) was over the trash can when control switched to it, causing the selected item to nearly be deleted accidentally.

Positioning Elements: Five of the six participant pairs frequently revisited already placed items to make small positional changes. These small positional changes were - in the majority of cases - aimed at aligning items placed early in the process with items placed recently. The changes were also a consequence of participants finding it difficult to finely acquire the target location, especially early on. One reason for this is that there is a small delay between the finger moving on the table and the selected item following this movement. Participants took a while to learn to compensate for this delay.

Order of Elements: Participants overwhelmingly placed the map view element first. This element was naturally the largest, and typically the next element placed was the map key. After that, there was no detectable order to how the remaining elements were placed, although it is worth noting that participants typically delayed selecting elements for deletion until late in the experiment.

Static vs Dynamic Representation: Several of the map construction activities involved participants creating layouts for dynamic map applications. Three of the six participant pairs noted at several stages that they were confused over whether the target map was dynamic or static. This confusion was reinforced by the fact that the map view element was a single static image.

Our first design principle for future map construction tools is to afford the dynamic elements (such as the map view) with some limited dynamic behaviour within the tool, so that users can see the impact of tool and data placement as the view shifts over the larger map.

Deleting Items: MED supported deleting elements through a trash can metaphor. Participants did use this feature, although deletion was not easily reversible so at least one entailment of the metaphor was broken. The basket served the purpose of removing extraneous elements from the element pool, although did so at the cost of making some part of the screen unusable for placing elements. There was also the problem of accidentally deleting elements through incorrectly recognised touchs, as discussed earlier in this section.

Our second design principle is to not use a separate space for deleting items. Rather, elements could be grayed out and deactivated if not wanted so that they can't be accidentally moved, and don't distract from those elements that will be placed. It should be possible to reactivate elements through some other gesture, so as to support undoing of actions.

\section{Limitations}

Our collaborative user experiment was limited to six pairs of participants due to a large overhead in video recording and post processing each experiment. Future studies should expand the number of participants.

As well as this, the table did not directly support concurrent placement of elements by different people as the application only processed one touch at any given point in time. However, this was deliberate as we were interested more in how the pairs would reach agreement over how the map should be constructed through discussion around the table, rather than how pairs might multi-task to more quickly generate the map.

\section{CONCLUSIONS}

In summary, while running a user study on collaborative online map creation, we observed a number of issues around the tool's design and the use of the touch table. For example, since we wished to discourage concurrent multi-tasking in the map creation (and instead foster discussion amongst the participant pairs) we made the application single-touch rather than multi-touch. This caused a few issues though when users naturally tried to override our design and simultaneously touched the table. Similarly, our creation of a trash can to allow pairs to remove visual clutter from the display also caused problems around retrieving items, as well as combining with the previous problem to create cases where items were trashed due to a stray finger causing an accidental touch at an inopportune moment.

We plan to publish the actual findings regarding the map element placing in an appropriate GIS publication venue once we complete a more detailed analysis of our results. Our hope for this paper is that it leads other user interface researchers towards better user studies involving collaboration around touch tables and map interface designs.

\section{REFERENCES}

1. E. M. Aoidh, M. Bertolotto, and D. C. Wilson. Understanding geospatial interests by visualizing map interaction behavior. Information Visualization, 7(3):275-286, 2008.

2. T. Apted, A. Collins, and J. Kay. Heuristics to support design of new software for interaction at tabletops. In CHI '09 Workshop on Multitouch and Surface Computing, 2009.

3. P. Isenberg. Collaborative Information Visualization in Co-located Environments. PhD thesis, University of Calgary, 2009.

4. J. Nielsen and K. Pernice. Eyetracking Web Usability. New Riders Press, 2009.

5. J. Schöning, F. Daiber, A. Krüger, and M. Rohs. Using hands and feet to navigate and manipulate spatial data. In Proceedings of the International Conference Extended Abstracts on Human Factors in Computing Systems (CHI), pages 4663-4668. ACM, 2009.

6. S. D. Scott, M. S. T., Carpendale, and K. M. Inkpen. Territoriality in collaborative tabletop workspaces. In Proceedings of the Conference on Computer Supported Cooperative Work (CSCW), pages 294-303. ACM, 2004.

7. D. Valkov, F. Steinicke, G. Bruder, and K. H. Hinrichs. Navigation through geospatial environments with a multi-touch enabled human-transporter metaphor. In Proceedings of Geoinformatik, 2010. 